\section{A New Look at the LDRT treatment for COVID-19 Associated Pneumonia: The Issues of Antiviral Resistance and Virus Spread-Ability}

\author{
Bevelacqua J. J. ${ }^{10}$, Mehdizadeh A. R. ${ }^{2}$, Mortazavi S. A. R. ${ }^{3}$, \\ Mortazavi S. M. J. $2,4 * \bullet$
}

$\mathrm{T}$ he rapid spread of COVID-19, a disease caused by the novel coronavirus (SARS-CoV-2) raises the key questions of whether the evolution of virus is driven by mutations [1]. We have previously addressed our concerns about the evolutionary mutations of the SARS$\mathrm{CoV}-2$ caused by putting a selective pressure on this virus [2-6]. Grubaugh et al., have also recently addressed the importance of these mutations in spreading this disease [7].

Although we and many of our colleagues do not believe that the so called linear non-threshold (LNT) hypothesis is a valid model for dose-response of ionizing radiation, we don't follow them in criticizing Hermann Joseph Muller (1926-1926) since our knowledge of radio-microbiology has been influenced by the findings of this scientist. With this introduction, Mortazavi et al., came across an article by Harris et al., several years ago in which a team of researchers used a source of Co-60 in a series of experiments to irradiate Escherichia coli with a dose that only left $1 \%$ of bacteria [8]. The experiment conducted by Harris et al., was an attempt to make this bacterium resistant to radiation. Obviously, they needed to increase the radiation dose each time to achieve the same survival fraction of 1 percent. Concerns about conducting such experiments at the time prompted them to publish a short paper in the IJPH highlighting the potential dangers of this type of radiation resistance that was caused through directed evolution [9]. The main philosophy of this paper was that the extremophile bacteria generated in such experiments would not only be highly resistant to radiation, but would possibly be resistant to all potential life-threatening factors for that microorganism, ranging from desiccation, UV and heat to antibiotics. Needless to say, even in an ideal circumstance, there would be a possibility of so-called "bacterial escape" from the laboratory. A laboratory release of an antibiotic-resistant extremophiles (AREs) could have significant consequences.

Low Dose Radiation Therapy for COVID-19 Associated Pneumonia

Low-dose radiation therapy (LDRT) was historically used to treat pneumonia during the first half of the $20^{\text {th }}$ century. In the aftermath of the COVID-19 pandemic, this concept was modified and introduced for treatment of COVID-19 associated pneumonia in March 2020 [10]. Moreover,
${ }^{1} \mathrm{PhD}$, Bevelacqua Resources, Richland, WA 99352, USA

${ }^{2} \mathrm{PhD}$, Department of Medical Physics and Engineering, School of Medicine, Shiraz University of Medical Sciences, Shiraz, Iran

${ }^{3} \mathrm{MD}$, School of Medicine, Shiraz University of Medical Sciences,

Shiraz, Iran

${ }^{4} \mathrm{PhD}$, lonizing and

Non-ionizing Radiation

Protection Research

Center (INIRPRC), Shiraz

University of Medical Sci-

ences, Shiraz, Iran

*Corresponding author:

S. M. J. Mortazavi

Department of Medical

Physics and Engineering

School of Medicine, Shi-

raz University of Medical

Sciences, Shiraz, Iran

E-mail: mortazavismj@ gmail.com

Received: 30 July 2020 Accepted: 7 August 2020 
scientists from Canada, Spain, United States, Germany and France have also confirmed the potential efficacy of LDRT for treatment of COVID-19 pneumonia. The rationale behind using LDRT as an effective treatment method for pneumonia in COVID-19 patients is mainly due to anti-inflammatory effects of LDR. Moreover, LDRT can combat the cytokine storm and reduce or prevent the risk of thrombosis.

\section{The Issue of Selective Pressure}

We believed that SARS-CoV-2, contrary to the claims of some experts, would be able to increase its pathogenicity and virulence through evolutionary processes. In view of this key point, we discussed that health care providers around the world are using a wide range of antiviral drugs. The use of these agents would exert a strong selective pressure on the virus that drives it toward further evolutionary mutations, mutations that enables the virus to spread much faster $[5,10]$. It is important to note that the predominant SARS-CoV-2 strain in Europe and the United States is now the G614, which has a much higher spread-ability than the previous strain, the D614. As an analogy to the use of antiviral drugs, health managers around the world are encouraging a person whose body is on fire to run, and this running only provides more oxygen to feed and expand the fire. The publication of our $1{ }^{\text {st }}$ paper on this issue "COVID-19 tragic pandemic: concerns over unintentional "directed accelerated evolution" of novel Coronavirus (SARS-CoV-2) and introducing a modified treatment method for ARDS" [4] as well as our subsequent papers [2, 3, 5] were indeed warnings of this danger.

As is clear from the definition of selective pressure, any factor that threatens the reproduction and life of an organism may causes a significant selective pressure "Selective pressure is any phenomena which alters the behavior and fitness of living organisms within a given environment. It is the driving force of evolution and natural selection, and it can be divided into two types of pressure: biotic or abiotic". For the virus, because today they are thought of as being in a gray area between living and nonliving, any intervention that endangers the activity of a virus, can exert such a selective pressure. Obviously, when radiation doses of tens of thousands of Grays are needed to inactivate a virus, a small radiation dose ( $\sim$ Gy or less) would only serve as a tickling dose. Given this consideration, we discussed that in contrast with low dose radiation (LDR), using antivirals poses a threat to virus activity and hence increases the chance of the viral evolution through mutations. Our recommended treatment, low dose radiation therapy, was a "symptom treatment" and it could be introduced as a "virus-friendly" treatment!

An important feature of SARS-CoV-2 is the protein spikes which cover its surface. The virus uses protein spikes to bind with and enter human cells. New studies indicate that evolutionary processes have enabled SARS-CoV-2 to have more stable spikes that binds 3 orders of magnitude stronger to human cells "These differences mean the spike of SARS-CoV-2 is more stable and is able to bind around 1,000 times more tightly to a human cell than this bat virus."

Grubaugh et al., in their paper published recently in Cell [7] state that based on the findings of Korber et al., [11] an amino acid change in the spike protein of SARS-CoV-2, D614G, has led to significant spread of viruses containing G614 in many places (Figure 1). However, Grubaugh et al., believe that the impact of this mutation on factors such as transmission, disease, and vaccine and therapeutic development are still mostly unknown.

\section{Mutations and Resistance against Antiviral Drugs}

Scientists believe that the remarkable rate of transmission and infectivity of the SARS-CoV-2 could be due to mutations making SARS-CoV-2 different from the earlier SARS-CoV virus'SARSCoV-2 shares homology with the SARS-CoV but the rate of transmission and infectivity of the 


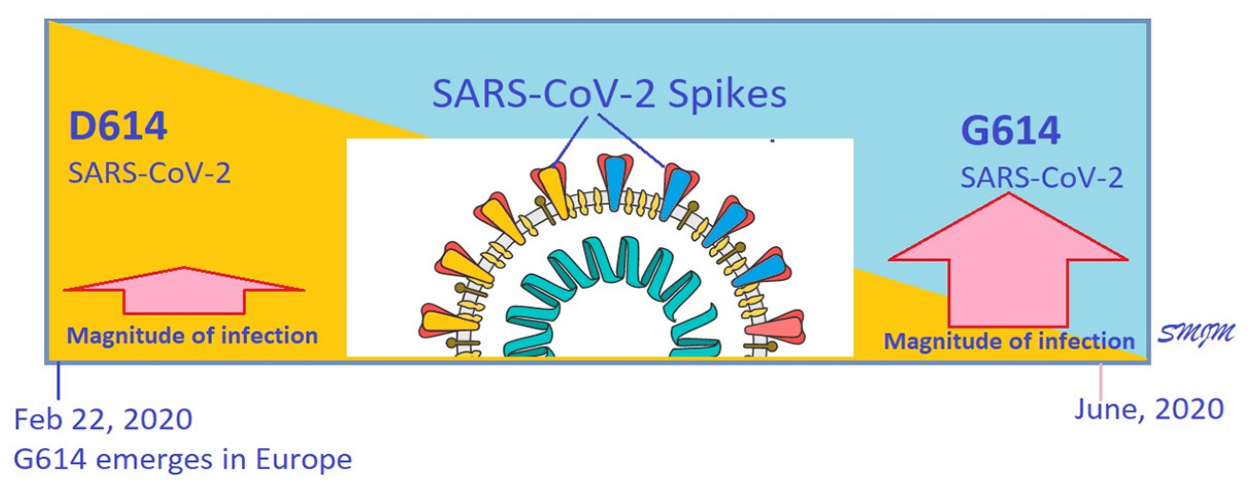

Figure 1: At the present time, more SARS-CoV-2 viruses circulating in the human population globally have the G614 form of the spike protein versus the D614 form that was originally identified people in Wuhan, China. (Figure originally from Korber et al., [11] and modified from a SARS-CoV-2 figure provided from Desiree Ho for the Innovative Genomics Institute. https://innovativegenomics.org/free-covid-19-illustrations/).

SARS-CoV-2 has been remarkable; this accelerated spreading rate may be due to a gain of function mutation, making this novel virus different from the SARS-CoV virus" [12].

Helmi et al., [13] state "Generally, coronaviruses are not sensitive to current antiviral drugs" and now we know that when the treatment is not effective and some genomes can replicate, selective pressures may result in rapid adaptation leading to viral resistance "Prolonged exposure to antiviral drugs and ongoing viral replication are key factors in the development of drug resistance. In cases where the treatment course is effective and viral fitness is impaired sufficiently, no viral genomes will be successfully replicated. However, in conditions where the treatment is not as effective and some genomes replicate, selective pressures may result in rapid adaptation leading to viral resistance. Antiviral resistance is linked to high mutation and recombination rates, demographic histories of transmission, compartmentalization, and selective forces incurred during viral adaptation to drugs" [14].

Again, regarding the key importance of SARS-CoV-2 mutations, it is worth noting that the antiviral drug "remdesivir" has recently been introduced as a standard of care for patients with COVID-19, as Anthony Fauci, has addressed it "will be the standard of care". However, whispers of resistance of some strains of the virus to this drug can be heard "Researchers have identified that coronaviruses sometimes carry a mutation against remdesivir, a drug that is currently under preclinical development and in clinical trials for treatment of COVID-19".

Although Ghadimi-Moghadam et al., first raised a red flag about the dangers of wide use of antiviral drugs for treatment of COVID-19, recent reports clearly confirm the role of wide use of antiviral drugs on exerting a strong selective pressure on the virus "other promising drugs, such as remdesivir, directly target the virus itself. Previous studies found two mutations in the enzyme gene that confer resistance to remdesivir, but the LSHTM study didn't find many instances of these mutations. Wide use of the drug, however, will put selective pressure on the virus (environmental factors that contribute to evolutionary change), so monitoring these mutations will be important" [15].

And more interestingly, scientists are still looking to use antiviral cocktails to solve the abovementioned problem, ignoring the fact that the virus can quickly become resistant to new antivirals "Viruses that carry remdesivir resistance mutations are actually more susceptible to EIDD-1931 and vice versa, suggesting that the two drugs could be combined for greater efficacy and to prevent the emergence of resistance,"said George Painter, PhD, executive officer of DRIVE and director 


\section{of the Emory Institute for Drug Development'.}

This note suggests that caution be used in prescribing antiviral drugs and other agents in the treatment of SARS-COV-2. These treatment methods could facilitate mutations that make SARS-COV-2 more aggressive and virulent. The use of low dose radiation has many advantages to disease treatment without the negative features of antiviral drugs. As such, low dose radiation represents an effective tool for SARS-COV-2 treatment and merits further study and clinical trials.

\section{Conflict of Interest}

None

\section{References}

1. Phan T. Genetic diversity and evolution of SARS-CoV-2. Infection, Genetics and Evolution. 2020;81:104260. doi: 10.1016/j.meegid.2020.104260.

2. Bevelacqua JJ, Mortazavi SAR, Mortazavi SMJ. Re: Low-dose radiation therapy for COVID-19 pneumonia: is there any supportive evidence? Int J Radiat Biol. 2020;1-2. doi: 10.1080/09553002.2020.1797213.

3. Mortazavi SMJ, Kefayat A, Cai J. Low-dose radiation as a treatment for COVID-19 pneumonia: A threat or real opportunity? Med Phys. 2020. doi: 10.1002/mp.14367.

4. Ghadimi-Moghadam A, Haghani M, Bevelacqua JJ, et al. COVID-19 Tragic Pandemic: Concerns over Unintentional "Directed Accelerated Evolution" of Novel Coronavirus (SARS-CoV-2) and Introducing a Modified Treatment Method for ARDS. J Biomed Phys Eng. 2020;10(2):241-6. doi: 10.31661/jbpe.v0i0.2003-1085. PubMed PMID: 32337192. PubMed PMCID: PMC7166223.

5. Mehdizadeh AR, Bevelacqua JJ, Mortazavi SAR, Mortazavi SMJ. COVID-19: Introducing Low Dose Radiation as an Effective Treatment for Pneumonia that Shouldn't Induce Selective Pressure and New Mutations. J Biomed Phys Eng. 2020;10(3):247-50. doi: 10.31661/jbpe.v0i0.2005-1114. PubMed PMID: 32637368. PubMed PMCID: PMC7321390.

6. Welsh JS, Bevelacqua JJ, Mozdarani H, Mortazavi SAR, Mortazavi SMJ. Why can COVID-19 fatality in space be significantly higher than on Earth? Int J Radiat Res. 2020;18(3):421-6. doi: 10.18869/acadpub.ijrr.18.3.421.

7. Grubaugh ND, Hanage WP, Rasmussen AL. Making Sense of Mutation: What D614G Means for the COVID-19 Pandemic Remains Unclear. Cell. 2020. doi: 10.1016/j.cell.2020.06.040.

8. Harris DR, Pollock SV, Wood EA, et al, Eggington J, Durfee TJ, Middle CM. Directed evolution of ionizing radiation resistance in Escherichia coli. J Bacteriol. 2009;191(16):5240-52. doi: 10.1128/JB.00502-09. PubMed PMID: 19502398. PubMed PMCID: PMC2725583.

9. Mortazavi SAR, Khademi F, Motamedifar M, Haghani M, Mortazavi SMJ. Human-Induced Radioresistance as a Possible Mechanism for Producing Biological Weapons: A Feasible Bridge between Radioresistance and Resistance to Antibiotics and Genotoxic Agents. Iranian Journal of Public Health. 2014;43(2):247-8.

10. Ghadimi-Moghadam A, Haghani M, Bevelacqua JJ, et al. COVID-19 tragic pandemic: concerns over unintentional "directed accelerated evolution" of novel Coronavirus (SARS-CoV-2) and introducing a modified treatment method for ARDS. J Biomed Phys Eng. 2020;10(2):241-6. doi: 10.31661/jbpe.v0i0.2003-1085. PubMed PMID: 32337192. PubMed PMCID: PMC7166223.

11. Korber B, Fischer WM, Gnanakaran S, Yoon H, et al. Tracking changes in SARS-CoV-2 Spike: evidence that D614G increases infectivity of the COVID-19 virus. Cell. 2020. doi: 10.1016/j.cell.2020.06.043.

12. Vellingiri B, Jayaramayya K, lyer M, Narayanasamy A, et al. COVID-19: A promising cure for the global panic. Science of the Total Environment. 2020;725:138277. doi: 10.1016/j.scitotenv.2020.138277.

13. Helmy YA, Fawzy M, Elaswad A, et al. The COVID-19 pandemic: a comprehensive review of taxonomy, genetics, epidemiology, diagnosis, treatment, and control. J Clinical Med. 2020;9(4):1225. doi: 10.3390/jcm9041225. PubMed PMID: 32344679 . PubMed PMCID: PMC7230578.

14. Gelman R, Bayatra A, Kessler A, Schwartz A, Ilan Y. Targeting SARS-CoV-2 Receptors as a Means for Reducing Infectivity and Improving Antiviral and Immune Response: An Algorithm-based Method For Overcoming Resistance To Antiviral Agents. Emerg Microbes Infect. 2020;9(1):1397-406. doi: 10.1080/22221751.2020.1776161. PubMed PMID: 32490731.

15. Crossan C. Mutating coronavirus: what it means for all of us. Medical Xpress, 2020. [Online]. Available from: https://medicalxpress.com/news/2020-06-mutating-coronavirus.html. 